\title{
Coleção Zoológica: uma abordagem científica para o ensino sobre artrópodes em uma escola pública do interior do Rio Grande do Sul, Brasil
}

\author{
Zoological Collection: a scientific approach to teaching about arthropods in a public school in the \\ interior of Rio Grande do Sul, Brazil \\ Colección Zoológica: una aproximación científica a la enseñanza de artrópodos en una escuela \\ pública del interior de Rio Grande do Sul, Brasil
}

Recebido: 18/05/2021 | Revisado: 27/05/2021 | Aceito: 28/05/2021 | Publicado: 12/06/2021

Karine Gehrke Graffunder
ORCID: https://orcid.org/0000-0001-8860-9889
Universidade Federal de Santa Maria, Brasil
E-mail: kagraffunder@gmail.com
Cíntia Moralles Camillo
ORCID: https://orcid.org/0000-0003-2876-9156
Universidade Federal de Santa Maria, Brasil
E-mail: cintiacamillo@gmail.com
Fabiana Lasta Beck Pires
ORCID: https://orcid.org/0000-0002-9556-2983
Instituto Federal de Educação, Ciência e Tecnologia Farroupilha, Brasil
E-mail: fabiana.pires@iffarroupilha.edu.br
Gerson Azulim Müller
ORCID: https://orcid.org/0000-0003-0342-4733
E-mail: gecoazul@hotmail.com

\section{Resumo}

As atividades práticas são recursos importantes para o ensino de Ciências, entretanto, a escassez de materiais biológicos impede sua ampla utilização na Educação Básica. Diante desse contexto, este estudo tem por objetivo analisar a contribuição da produção de uma coleção zoológica ao processo de ensino e aprendizagem sobre artrópodes em uma turma com 16 alunos do $7^{\circ}$ ano de uma escola pública do interior do Rio Grande do Sul. Trata-se de uma pesquisa de laboratório estabelecida através de uma abordagem teórico-prática inicial do conteúdo sobre artrópodes, com posterior confecção coletiva de uma coleção zoológica de artrópodes (abrangendo processos de coleta, montagem e conservação). A análise dos resultados ocorreu por meio de observação e registro em diário de campo. Se obteve 16 exemplares do subfilo Chelicerata, sete espécimes do Myriapoda, dois exemplares do Crustacea e 38 espécimes do Hexapoda. A coleção se mostrou um material eficiente para o processo de ensino sobre artrópodes, uma vez que, tal ferramenta é de baixo custo e com potencialidade de atrair o interesse e a curiosidade dos alunos. Além disso, aproxima os estudantes do conteúdo desenvolvido em sala de aula, auxiliando no entendimento sobre aspectos morfológicos, anatômicos, fisiológicos e ecológicos. Ademais, conclui-se que permitiu sinalizar diferenças e esclarecer mitos, contribuindo para uma aprendizagem significativa e a ser utilizado em aulas futuras.

Palavras-chave: Caixa entomológica; Educação básica; Ensino de ciências; Recurso didático; Zoologia de invertebrados.

\begin{abstract}
Practical activities are important resources for science teaching, however, the scarcity of biological materials prevents their widespread use in Basic Education. In this context, this study aims to analyze the contribution of the production of a zoological collection to the process of teaching and learning about arthropods in a class with 16 students from the 7 th year of a public school in the interior of Rio Grande do Sul. It is a laboratory research established through an initial theoretical-practical approach to the content of arthropods, with subsequent collective production of a zoological collection of arthropods (covering collection, assembly and conservation processes). The analysis of the results occurred through observation and recording in a field diary. We obtained 16 specimens of the subphylum Chelicerata, seven specimens of Myriapoda, two specimens of Crustacea and 38 specimens of Hexapoda. The collection proved to be an efficient material for the teaching process on arthropods, since such a tool is low cost and has the potential to attract students' interest and curiosity. In addition, it brings students closer to the content developed in the classroom, helping to understand morphological, anatomical, physiological and ecological aspects. In
\end{abstract}


addition, it is concluded that it allowed to signal differences and clarify myths, contributing to a meaningful learning and to be used in future classes.

Keywords: Entomological box; Basic education; Science teaching; Didactic resource; Invertebrate zoology.

\section{Resumen}

Las actividades prácticas son recursos importantes para la enseñanza de las ciencias, sin embargo, la escasez de materiales biológicos impide su uso generalizado en la Educación Básica. En este contexto, este estudio tiene como objetivo analizar la contribución de la producción de una colección zoológica al proceso de enseñanza y aprendizaje sobre artrópodos en una clase con 16 estudiantes de $7^{\circ}$ año de una escuela pública del interior de Rio Grande do Sul. Es una investigación de laboratorio establecida a través de una aproximación teórico-práctica inicial al contenido sobre artrópodos, con posterior producción colectiva de una colección zoológica de artrópodos (abarcando procesos de recolección, ensamblaje y conservación). El análisis de los resultados se realizó mediante observación y registro en un diario de campo. Obtuvimos 16 ejemplares del subfilo Chelicerata, siete ejemplares de Myriapoda, dos ejemplares de Crustacea y 38 ejemplares de Hexapoda. La colección demostró ser un material eficaz para el proceso de enseñanza sobre artrópodos, ya que dicha herramienta es de bajo costo y tiene el potencial de atraer el interés y la curiosidad de los estudiantes. Además, acerca al alumno a los contenidos desarrollados en el aula, ayudando a comprender aspectos morfológicos, anatómicos, fisiológicos y ecológicos. Además, se concluye que permitió señalar diferencias y esclarecer mitos, contribuyendo a un aprendizaje significativo y para ser utilizado en futuras clases.

Palabras clave: Caja entomológica; Educación básica; Enseñanza de las ciencias; Recurso didáctico; Zoología de invertebrados.

\section{Introdução}

A compreensão e aplicação da Ciência tem sido essencial para o desenvolvimento de um país, devido ao seu caráter social e econômico (Deboer, 2019). Contudo, no Brasil a cultura científica da população ainda é considerada baixa, o que dificulta a compreensão do mundo natural e seus fenômenos (Oliveira, 2017). Embora a contemporaneidade seja marcada pela avaliação e consolidação da popularização da Ciência, o Ensino de Ciências (EC) permanece voltado para práticas conteudistas, memorização de dados e aplicação de regras científicas (Anjos \& Carbo, 2019; Deboer, 2019).

Pereira (2014) defende que a educação e o trabalho docente são ferramentas de apoio na formação do cidadão, situado no novo mundo informatizado e globalizado. Para Chassot (2003) a alfabetização científica (AC) pode ser considerada como uma das dimensões para potencializar alternativas que privilegiam uma educação mais comprometida, com uma preocupação maior em enfatizá-la no Ensino Fundamental. Diante desse contexto, a Ciência também precisa ser lida e interpretada.

Segundo Lorenzetti e Delizoicov (2000), existem três tipos de AC: a alfabetização científica prática, que permite ao indivíduo resolver de forma imediata problemas básicos do cotidiano; a alfabetização científica cívica, que torna o cidadão mais atento e comprometido às ciências e seus impactos, e, por último, a alfabetização científica cultural, que em geral, é destinada à pequena parcela da população que se interessa por saber sobre ciências de maneira mais aprofundada. De modo prático, a AC permite conciliar uma leitura dinâmica do mundo e da palavra, que garanta ao sujeito uma organização lógica do pensamento e uma visão crítica (Graffunder, Camillo, Oliveira \& Goldschmidt, 2020). Para Marques e Marandino (2018), permitir aos alunos refletir e solucionar questões científicas implica na necessidade de articular novas metodologias de ensino, que os desafiem, estimulem e integrem.

No campo de Ciências Biológicas, a Zoologia tem grande destaque no currículo das escolas primárias e secundárias no Brasil, dedicando-se ao estudo dos animais (Matos, Oliveira, Santos \& Ferraz, 2009). O filo Arthropoda é um grupo diverso, abrangendo animais dotados de exoesqueleto e pernas articuladas com importância ecológica, médica e econômica. Por exemplo, os insetos (Hexapoda), que pertencem ao filo Arthropoda, estão envolvidos em processos ecológicos, como a polinização, o ciclismo de nutrientes e a cadeia alimentar, além de aspectos da parasitologia e pragas agrícolas (Borror \& Delong, 1988). Porém, a complexidade e a quantidade de conteúdos trazem desafios para o seu entendimento.

As coleções zoológicas para fins didáticos possibilitam a observação detalhada da morfologia e anatomia dos animais, ampliando a reflexão crítica sobre a conservação da biodiversidade (Tonini, Sarmento-Soares, Roldi \& Lopes, 2016). As 
coleções zoológicas concedem suporte de informação para análises da biodiversidade, ecologia, filogenia e evolução das espécies, sendo confeccionadas a partir de espécimes, ou suas partes, e mantidas em um ambiente com condições artificiais (Santos et al. 2021).

O uso de coleções zoológicas em aulas de ciências auxilia na compreensão das características anatômicas e morfológicas dos artrópodes, esclarecendo e diminuindo o frequente senso comum de que esse grupo envolve apenas organismos venenosos que causam doenças e prejuízos (Leon et al. 2015). Além disso, a elaboração de coleções zoológicas, especialmente de artrópodes, mostra-se fácil, de baixo custo e não necessita de laboratórios de ciências sofisticados (Wegener, Wesner, Moraes \& Müller, 2016). Diante desse contexto, este estudo tem por objetivo analisar a contribuição da produção de uma coleção zoológica ao processo de ensino e aprendizagem sobre artrópodes em uma turma do $7^{\circ}$ ano de uma escola pública do interior do Rio Grande do Sul.

\section{Metodologia}

Uma pesquisa pode ser classificada em relação à sua natureza, aos objetivos, à abordagem do problema e aos procedimentos técnicos adotados (Gil, 2010). De natureza aplicada, esta pesquisa objetiva gerar conhecimentos a partir de uma aplicação prática e dirigida. Quanto aos objetivos, é definida como exploratória, uma vez que proporciona uma nova visão ao problema.

O procedimento técnico adotado para este artigo é a Pesquisa de Laboratório (PL). Marconi e Lakatos (2017) consideram a PL como um procedimento difícil, contudo possibilita que o pesquisador controle a ação. Ainda, segundo as autoras, esta técnica deve ser planejada e relacionada com o objeto de estudo, ocorrendo geralmente em laboratórios, na sala de aula ou ao ar livre. É importante destacar que o pesquisador pode mesclar os ambientes, a fim de atingir o seu objetivo.

Quatro aspectos devem ser levados em consideração: objeto, objetivo, instrumental e técnicas. Especificamente, os objetos de estudo podem ser pessoas ou animais, vegetais ou minerais. Na pesquisa de laboratório com pessoas, estas são colocadas em ambientes controlados pelo pesquisador, que efetua a observação sem tomar parte pessoalmente (Marconi \& Lakatos, 2017, p.83).

No que se refere ao Ensino de Ciências, a PL proporciona ao pesquisador observar, pontuar e registrar. Perante ao exposto, o estudo foi realizado com 16 alunos (13 do sexo masculino e três do sexo feminino), com idades entre 11 e 14 anos de uma turma de $7^{\circ}$ ano do Ensino Fundamental de uma Escola Estadual de Ensino Público no município de Condor, RS. A identidade dos sujeitos participantes da pesquisa foi mantida em sigilo e identificados na análise por letras do alfabeto, bem como a professora regente da turma.

\subsection{Abordagem teórico-prática}

Para o desenvolvimento da atividade de elaboração da coleção zoológica, um conjunto de atividades sobre o tema "Artrópodes" foi realizada, a fim de introduzir e ampliar os conhecimentos teóricos dos alunos sobre o assunto. As atividades foram elaboradas a partir de quatro objetivos específicos: (1) Motivar os alunos a participarem da construção de uma coleção zoológica; (2) Desenvolver a construção de valores e habilidades como respeito e cooperação em grupo; (3) Aprimorar a capacidade de relacionar e sistematizar conceitos; e (4) Entender a classificação e as diferenças dos subfilos Chelicerata, Myriapoda, Crustacea e Hexapoda. A abordagem dos conceitos ocorreu por meio de atividades diversas, conforme o Quadro 1. 
Quadro 1. Descrição da série de atividades.

\begin{tabular}{|c|c|c|}
\hline Aula & Conteúdos & Atividades \\
\hline 1 & $\begin{array}{l}\text { Artrópodes: Características gerais, adaptações evolutivas e } \\
\text { classificação }\end{array}$ & $\begin{array}{c}\text { Aula expositiva dialogada } \\
+ \\
\text { Resolução de exercícios }\end{array}$ \\
\hline 2 & $\begin{array}{l}\text { Chelicerata: Ordem Aranae e Ordem Scorpionida } \\
\text { Estrutura corporal, digestão, excreção e reprodução }\end{array}$ & Produção de desenhos \\
\hline 3 & $\begin{array}{l}\text { Chelicerata: Ordem Opiliones e Ordem Acari } \\
\text { Estrutura corporal e características em geral }\end{array}$ & $\begin{array}{c}\text { Elaboração de } \\
\text { Mapas Conceituais }\end{array}$ \\
\hline 4 & $\begin{array}{c}\text { Myriapoda: Classe Chilopoda e Diplopoda } \\
\text { Estrutura corporal } \\
\text { Crustacea: } \\
\text { Características gerais e exemplos }\end{array}$ & $\begin{array}{c}\text { Pesquisa }+ \\
\text { Estudo dirigido }\end{array}$ \\
\hline 5 & $\begin{array}{c}\text { Revisão Geral: } \\
\text { Confecção de modelos tridimensionais sobre os Chelicerata, } \\
\text { Myriapoda e Crustacea }\end{array}$ & Aula prática I \\
\hline 6 & $\begin{array}{c}\text { Hexapoda: } \\
\text { Características gerais, desenvolvimento e excreção }\end{array}$ & $\begin{array}{l}\text { Aula expositiva dialogada/ } \\
\text { Estudo dirigido }\end{array}$ \\
\hline 7 & $\begin{array}{c}\text { Filo Arthropoda: } \\
\text { Importância médica, econômica e ecológica }\end{array}$ & $\begin{array}{l}\text { Elaboração de cartazes + } \\
\text { Socialização }\end{array}$ \\
\hline 8,9 e 10 & $\begin{array}{c}\text { Revisão Geral: } \\
\text { Coleção Zoológica de Artrópodes: coleta, montagem e } \\
\text { conservação }\end{array}$ & Aula prática II \\
\hline
\end{tabular}

Fonte: Autores.

A confecção da coleção zoológica ocorreu ao final da série de atividades, uma vez que foi organizada para ser aplicada após os estudantes receberem o suporte teórico necessário para execução da proposta. As explicações estabelecidas envolveram aspectos evolutivos de cada grupo, diferenças morfológicas e a sua importância ecológica, médica e econômica, conforme os pressupostos estabelecidos por Hickman, Roberts e Keen (2016).

A construção do recurso didático com os alunos teve por finalidade desenvolver a capacitação na coleta, manipulação e no acondicionamento de material biológico; e incentivar a busca de informações e identificação dos artrópodes. Diante desse pressuposto, segue-se a descrição sobre o processo completo da construção da coleção zoológica de artrópodes.

\subsection{Confecção da coleção zoológica de artrópodes}

A construção da coleção é composta de três etapas denominadas: coleta, montagem e conservação, ou seja, três ações didáticas condutoras. $\mathrm{O}$ tempo total de sua aplicação em aula corresponde a 6 períodos/aula de 50 minutos.

\subsubsection{Coleta}

Esta etapa teve por finalidade aprimorar a capacidade de organização de materiais pelos alunos e na divisão de tarefas, assim como, desenvolver o trabalho em equipe. O tempo estipulado para a explicação das tarefas desta fase foi de duas horas. Nesse contexto, inicialmente os alunos foram divididos em grupos com número máximo de quatro alunos, a fim de coletarem exemplares diversos pertencentes ao grupo dos artrópodes (Figura 1). Solicitou-se que os grupos obrigatoriamente realizassem a coleta de um espécime de cada grupo e trouxessem na próxima aula.

O processo de coleta/conservação dos espécimes em casa e aspectos de segurança foram esclarecidos aos alunos em aula, junto de uma demonstração no jardim da escola com o uso de potes plásticos, pinças e álcool (disponibilizados pelo colégio). De acordo com Almeida, Ribeiro-Costa \& Marinoni (2003), os exemplares de Chelicerata, Myriapoda e Crustacea 
são mortos e acondicionados em frascos de vidro com álcool 70\% e os Hexapoda mortos no refrigerador, para posterior montagem a seco. Perante ao exposto, posteriormente os estudantes continuaram a tarefa em momento extraclasse (em casa) durante sete dias, sendo orientados para fazer essa atividade acompanhados de seus responsáveis.

Figura 1. Exemplos de cada grupo para coleta.

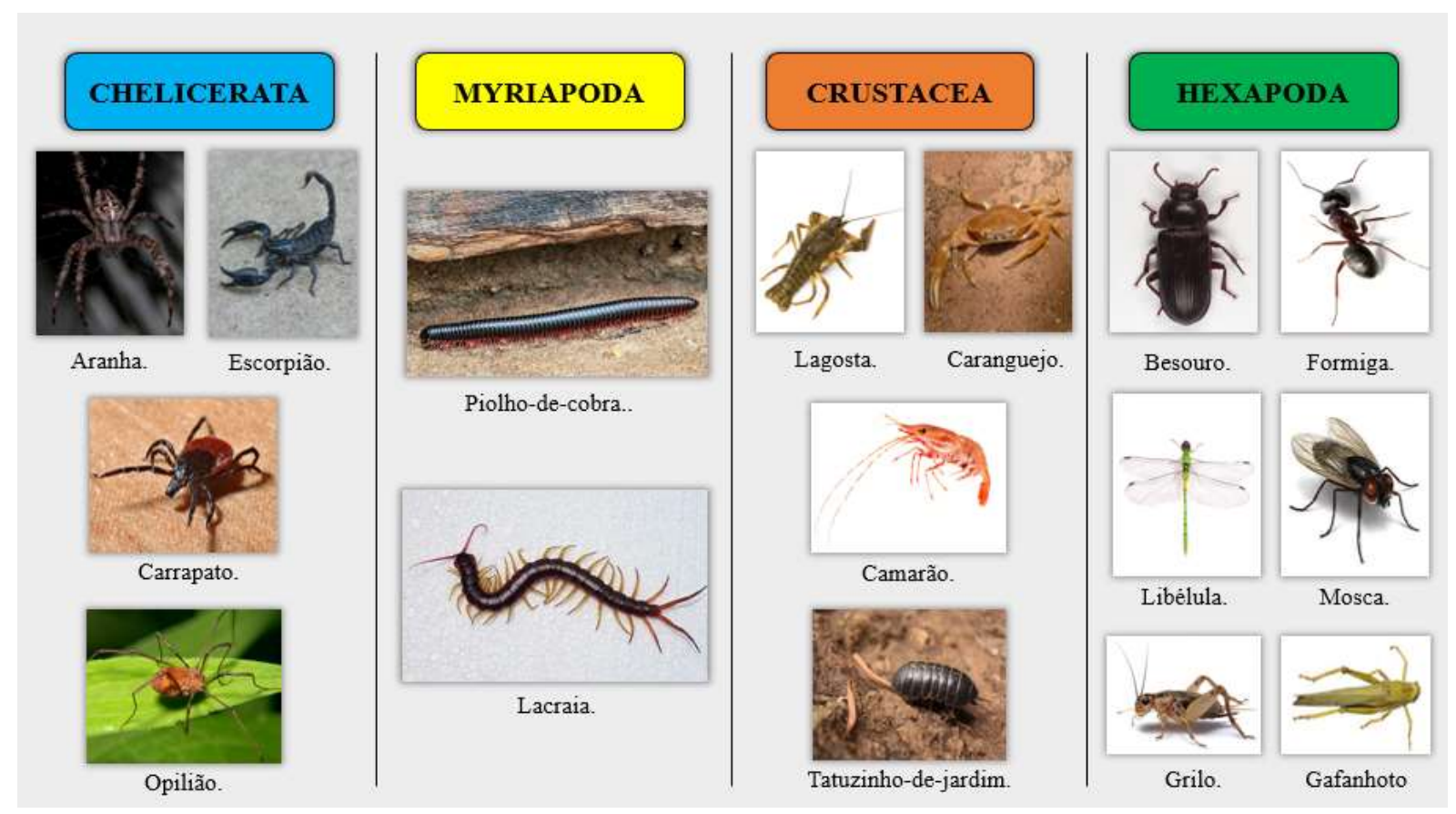

Ilustrações disponíveis em <https://pixabay.com/pt/>. Fonte: Autores.

\subsubsection{Montagem}

Esta etapa teve por objetivo motivar os alunos a participarem da construção da coleção zoológica, a fim de relacionar conceitos na aplicação prática do processo e desenvolver a cooperação em grupo. O período utilizado para o processo de montagem em aula foi de duas horas aula.

As formas de montagem foram adaptadas do "Manual de coleta, conservação, montagem e identificação de insetos" dos autores Almeida, Ribeiro-Costa e Marinoni (2003). Ilustrações de coleções zoológicas foram disponibilizadas para visualização, o que contribui na compreensão das tarefas a serem realizadas. Os recursos necessários para essa fase são: canetão, quadro, isopor, alfinetes, papeis, exemplares biológicos de artrópodes, naftalina, gase, caixa de papel, caneta, papel e fita. O material coletado e levado à escola pelos estudantes permaneceu no laboratório do colégio para execução completa da ação.

\subsubsection{Conservação}

A terceira etapa teve por finalidade revisar conceitos junto aos alunos, a fim de esclarecer dúvidas e estimular a socialização do material produzido pela turma. O período estipulado dessa tarefa em aula foi de duas horas.

Após a coleta e montagem dos exemplares pelos alunos, o material foi organizado para sua conservação. Os estudantes participaram da seleção e organização dos exemplares que compõem a coleção zoológica, que após sua confecção, foi doada à escola. O material obtido foi identificado e classificado de acordo com Santos, Silva e Antunes (2018), conforme o Quadro 2, nos quatro subfilos 'Chelicerata', 'Myriapoda', 'Crustacea' e 'Hexapoda' (classificação em filo, subfilo e nome popular). Os três primeiros grupos foram acondicionados em álcool 70\% e os Hexapoda foram armazenados em uma caixa, constituída de papel, isopor e naftalinas cobertas por gaze. 
Quadro 2. Critérios de categorização para identificar e classificar os espécimes coletados.

\begin{tabular}{|c|c|c|c|}
\hline $\begin{array}{c}\text { FILO } \\
\text { ARTHROPODA }\end{array}$ & Conteúdos & Número de patas & Antenas \\
\hline $\begin{array}{c}\text { Subfilo } \\
\text { Chelicerata }\end{array}$ & $\begin{array}{l}\text { Cefalotórax e abdome (aranha, escorpião e } \\
\text { carrapato). } \\
\text { Cefalotórax e abdome fundido (opilião). }\end{array}$ & 4 pares & Ausentes \\
\hline $\begin{array}{c}\text { Subfilo } \\
\text { Myriapoda }\end{array}$ & $\begin{array}{l}\text { Diplópodes: cabeça, tórax e abdome. } \\
\text { Exemplo: piolho-de-cobra. } \\
\text { Quilópode: } \\
\text { cabeça e tronco. } \\
\text { Exemplo: lacraia. }\end{array}$ & $\begin{array}{l}\text { Diplópodes: } 2 \text { pares por } \\
\text { segmento. } \\
\text { Quilópode: } 1 \text { par por segmento. }\end{array}$ & 1 par \\
\hline $\begin{array}{c}\text { Subfilo } \\
\text { Crustacea }\end{array}$ & Cefalotórax e abdome. & $\begin{array}{l}\text { Camarão, lagosta e caranguejo: } \\
5 \text { pares de pernas. } \\
\text { Tatuzinho-de-jardim: } 7 \text { pares de } \\
\text { pernas. }\end{array}$ & 2 pares \\
\hline $\begin{array}{c}\text { Subfilo } \\
\text { Hexapoda }\end{array}$ & Cabeça, tórax e abdome. & 3 pares & 1 par \\
\hline
\end{tabular}

Fonte: Autores.

Além disso, ressaltou-se que os artrópodes (Arthropoda, do grego arthro $=$ articulado + podos $=$ pés) são o grupo taxonômico mais diversificado, com distribuição por quase todo o globo e ocupando todos os habitats. São organismos invertebrados com exoesqueleto rígido (presença de quitina), apresentando apêndices articulados (patas e antenas) e de número diferenciado de acordo com o subfilo a que pertencem (Santos, Silva \& Antunes, 2018).

\subsection{Análise da contribuição do material produzido ao aprendizado dos alunos}

A análise ocorreu por meio de observação e registro em diário de campo, considerando-se os conhecimentos mobilizados pelos estudantes e a eficácia das atividades propostas ao final da abordagem didática.

\section{Resultados e Discussão}

A coleção zoológica final, elaborada e organizada pelos alunos, resultou em 16 exemplares do subfilo Chelicerata, sete espécimes do Myriapoda, dois exemplares do Crustacea e 38 espécimes do Hexapoda, gerando um total de 63 exemplares de artrópodes (Quadro 3). 
Research, Society and Development, v. 10, n. 7, e2610716248, 2021

(CC BY 4.0) | ISSN 2525-3409 | DOI: http://dx.doi.org/10.33448/rsd-v10i7.16248

Quadro 3. Número de exemplares obtidos em cada subfilo dos artrópodes.

\begin{tabular}{|c|c|c|}
\hline $\begin{array}{l}\text { Subfilos dos } \\
\text { artrópodes }\end{array}$ & Exemplares & Total \\
\hline Crustacea & 2 tatuzinhos-de-jardim & 2 \\
\hline Myriapoda & $\begin{array}{c}5 \text { piolhos-de-cobra } \\
2 \text { lacraias }\end{array}$ & 7 \\
\hline Chelicerata & $\begin{array}{c}8 \text { aranhas } \\
5 \text { escorpiões } \\
3 \text { opiliões }\end{array}$ & 16 \\
\hline Hexapoda & $\begin{array}{c}22 \text { besouros } \\
3 \text { grilos } \\
2 \text { (borboletas, gafanhotos, mariposas) } \\
1 \text { (abelha, barata, cigarra, formiga, libélula, mosquito) }\end{array}$ & 38 \\
\hline
\end{tabular}

Fonte: Autores.

A turma apresentou grande interesse durante o período de atividades (Figura 2). Além disso, a confecção da coleção (Figura 3) proporcionou o desenvolvimento de aspectos investigativos e criativos, articulando-se conceitos teórico-práticos sobre o grupo dos artrópodes.

Figura 2. Construção da coleção zoológica por alunos do $7^{\circ}$ ano.

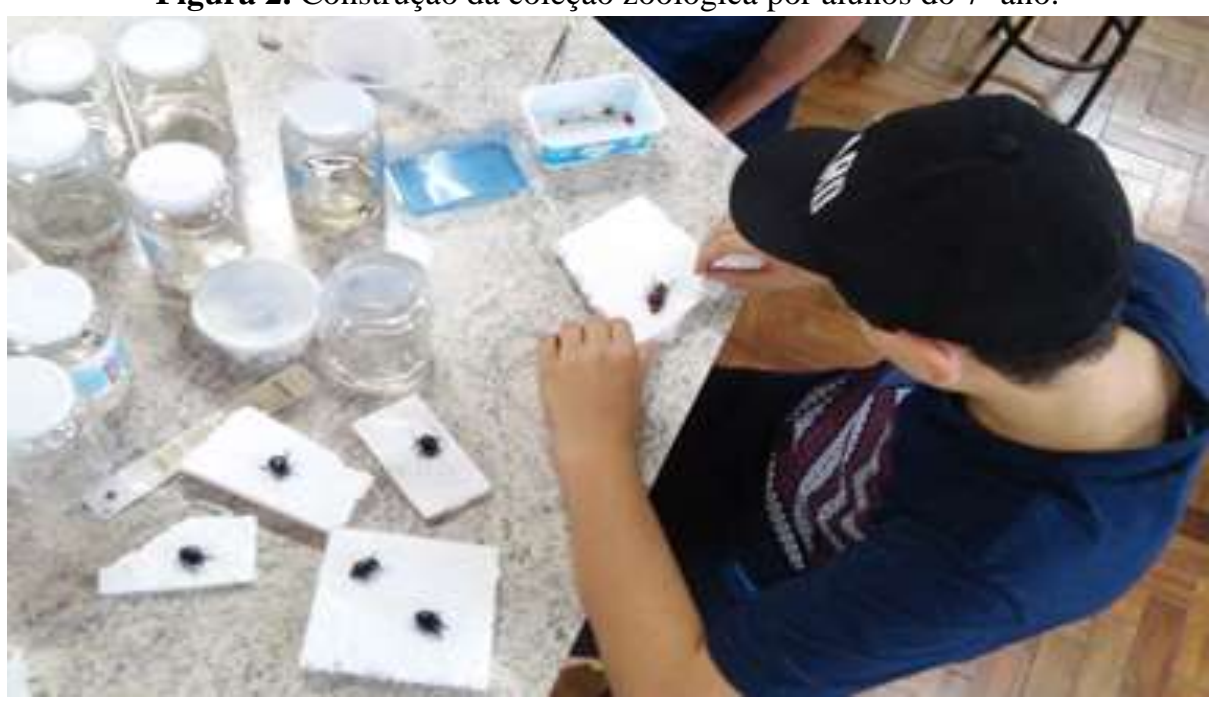

Fonte: Autores. 
Figura 3. Coleção zoológica finalizada e doada à escola pela turma do $7^{\circ}$ ano.

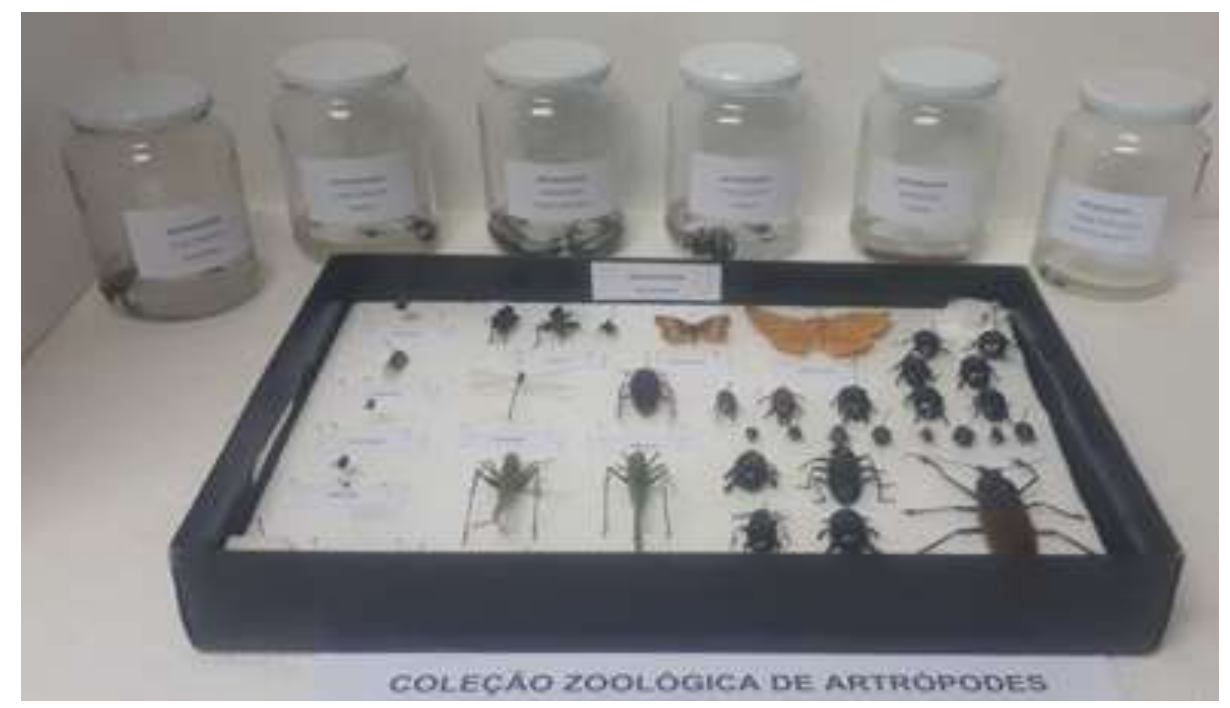

Fonte: Autores.

Conforme relato dos alunos e da professora regente da turma:

"Esse tipo de atividade auxiliou no entendimento sobre os artrópodes, principalmente sobre a segmentação corporal" (Aluna A).

"Aulas práticas com esses animais tornam mais fácil entender o que está presente e ausente em cada grupo" (Aluno $B)$.

"Esse tipo de atividade permitiu esclarecer dúvidas sobre como ocorre o processo de confecção de uma coleção zoológica" (Aluno C).

"A confecção das coleções aproxima os estudantes do conteúdo trabalhado em sala de aula, além de se constituir um importante recurso didático a ser utilizado em aulas futuras" (Professora A).

Para Papavero (1994, p.23) uma coleção didática contém material destinado ao ensino, demonstração e treinamento. O material didático pode ter curta duração, devido ao manuseio constante que ocasiona sua danificação. Para o autor, coleções didáticas são objetos de renovação permanente e completamente independentes das coleções de pesquisa, uma vez que as coleções podem receber espécimes parcialmente danificadas que podem servir para inúmeras finalidades didáticas.

A coleta de exemplares de aranhas e opiliões tornou possível sinalizar as diferenças entre ambos, já que são animais semelhantes e pertencentes ao mesmo subfilo Chelicerata. Desconhecido por alguns alunos, o opilião não apresenta constrição entre o cefalotórax e abdome, contando com a presença de um único par de olhos e glândulas odoríferas. Já a aranha apresenta corpo dividido em cefalotórax e abdome, além das estruturas denominadas quelíceras e pedipalpos. Os espécimes do subfilo Myriapoda (quilópodes e diplópodes) permitiram o esclarecimento quanto à peçonha, sendo a lacraia a qual apresenta forcípulas pela qual são injetados o veneno e o piolho-de-cobra toxinas que podem manchar a pele.

O número de besouros obtidos (30 exemplares), o que correspondeu a $60 \%$ do total de insetos coletados, deixou os alunos curiosos. Destacou-se assim, que apesar de sua fauna ser pouco conhecida, os besouros (Ordem Coleoptera) compõem 40\% das espécies conhecidas de insetos (em torno de 350.000 espécies descritas), com relevantes papéis no ecossistema, por exemplo, a decomposição de matéria orgânica e a polinização (Lewinsohn \& Prado, 2005).

A realização da coleção também permitiu a explicação de princípios evolutivos ligados à alta diversidade dos artrópodes (Hickman, Roberts \& Keen, 2016). Alguns fatores abordados foram a adaptação à vida terrestre, dispersão por meio 
do voo (presença e ausência de asas dos insetos), resistência a dessecação (devido a presença de quitina), órgãos sensoriais desenvolvidos, evolução conjunta às angiospermas (ligada a diversidade de nichos) e a importância do desenvolvimento por metamorfose, o que limita a competição intraespecífica.

Aspectos econômicos e ecológicos foram relacionados aos exemplares por meio de temáticas, como: abelhas e a produção de mel, artrópodes na alimentação humana, terapia larval, entomologia forense e insetos e suas doenças. A terapia larval pode ser utilizada para o tratamento de feridas cutâneas, especialmente em países e regiões de nível socioeconômico precário, por seu baixo custo e grande eficiência (Marcondes, 2006). Por sua vez, a entomologia forense contribui para elucidar uma série de crimes, como tráfico de entorpecentes, danos em imóveis e casos de contaminação (Pacheco \& Pereira, 2021).

Entomologia é a área da biologia que estuda os insetos (Hickman, Roberts \& Keen, 2016). Considerando a coleção entomológica como alternativa didática, Santos e Souto (2011) verificaram que essa atividade modificou a percepção que os alunos tinham sobre os insetos, os quais, habitualmente, são lembrados apenas por serem aqueles que invadem residências, transmitem doenças ou causam prejuízos. Em seu estudo, os autores constataram que a coleção entomológica se mostrou um recurso eficiente para o ensino de Entomologia no Ensino Fundamental, onde 75\% dos alunos obtiveram avanço cognitivo após sua utilização.

De acordo com Krasilchik (2004), cada situação exige uma solução própria, e atividades variadas podem atrair e interessar os alunos, atendendo às diferenças individuais. Os critérios metodológicos adotados estão relacionados de acordo com as atividades a serem realizadas em sala de aula, quais sejam: falar, fazer e mostrar. Sob essa perspectiva, a alfabetização científica se constitui uma contínua, que assim como a própria Ciência, deve estar sempre em construção, englobando novos conhecimentos pela análise e em decorrência de novas situações (Sasseron, 2015). Verifica-se que a atividade de confecção da coleção didática é capaz de despertar a curiosidade e um maior senso de crítico nos alunos, contribuindo para o "fazer ciência".

Por fim, há ainda muitas fragilidades na área educacional para serem questionadas e refletidas. Este trabalho buscou contribuir com o uso de uma estratégia prática no componente curricular de Ciências, proporcionando experiências que tornem o processo de ensino mais eficaz e a aprendizagem significativa. Nesse contexto, a formação do indivíduo deve ser estabelecida por um processo mediado, contínuo e de qualidade, o que requer materiais/espaços e metodologias adequadas.

\section{Considerações Finais}

Este estudo teve por objetivo analisar a contribuição da produção de uma coleção zoológica ao processo de ensino e aprendizagem sobre artrópodes em uma turma com 16 alunos do $7^{\circ}$ ano de uma escola pública do interior do Rio Grande do Sul. O material permitiu destacar a diversidade do grupo; sinalizar diferenças entre os exemplares coletados; e esclarecer a importância dos artrópodes no equilíbrio do ecossistema, desmistificando a ideia de que o grupo abrange apenas animais perigosos e que causam prejuízos. Além disso, aspectos econômicos e ecológicos foram articulados à construção da coleção, como os temas acerca das abelhas e a produção de mel; artrópodes na alimentação humana; terapia larval; entomologia forense e insetos e suas doenças.

A confecção da coleção zoológica de artrópodes se mostrou uma prática eficiente para a melhoria do processo de ensino sobre artrópodes no Ensino Fundamental, já que tal ferramenta constitui um material de baixo custo e com potencialidade de atrair o interesse dos alunos. Todavia, é flexível a mudanças e adaptações para diferentes níveis e modalidades de ensino, com atenção à classe a que se destina, do tempo e dos recursos disponíveis.

O material aproximou os estudantes do conteúdo trabalhado em sala de aula, além de se constituir um importante recurso didático a ser utilizado em aulas futuras. O processo de confecção da caixa despertou o senso crítico, a curiosidade e o empenho dos alunos pelo estudo, favorecendo uma aprendizagem significativa. 


\section{Referências}

Almeida, L. M. D., Ribeiro-Costa, C. S., \& Marinoni, L. (2003). Manual de coleta, conservação, montagem e identificação de insetos. In Manual de coleta, conservação, montagem e identificação de insetos (pp. 78-78).

Anjos, M. S., \& Carbo, L. (2019). Enfoque CTS e a atuação de professores de ciências. Enfoque CTS e a atuação de professores de Ciências. ACTIO, 4(3), 3557 .

Borror, D. J., \& DeLong, D. M. (1988). Introdução ao estudo dos insetos. Edgard Blucher. 653p.

Chassot, A. I. (2003). Alfabetização científica: uma possibilidade para a inclusão social. Revista Brasileira de Educação, 23(22), 89-100.

DeBoer, G. (2019). A history of ideas in science education. Teachers College Press.

Gil, A. C. (2010). Como elaborar projetos de pesquisa. (5a ed.), Atlas.

Graffunder, K. G., Camillo, C. M., Oliveira, N. M., \& Goldschmidt, A. I. (2020). Alfabetização científica e o ensino de Ciências na Educação Básica: panorama no contexto das pesquisas acadêmicas brasileiras nos últimos cinco anos de ENPEC. Research, Society and Development, 9(9), e313997122e313997122.

Hickman, C. P., Roberts, L. S., \& Keen, S. L. (2016). Princípios integrados de zoologia. Grupo Gen-Guanabara Koogan.

Krasilchik, M. (2004). Prática de ensino de biologia. Edusp.

León, R. G., González, G. M., Villacorta, Á. T., Rodríguez, C. D. P. S., Balam, F. C., Góngora, Á. R., \& Mejía, J. C. (2015). Aproximación y difusión de la Enfermedad de Chagas en dos comunidades de México por medio de colecciones entomológicas creadas con los estudiantes de primaria. Revista de Educación en biologia, 18(1), 79-87.

Lewinsohn, T. M., \& Prado, P. I. (2005). How many species are there in Brazil? Conservation Biology, 19(3), 619-624.

Lorenzetti, L., \& Delizoicov, D. (2000). Alfabetização científica no contexto das séries iniciais (Doctoral dissertation, Universidade Federal de Santa Catarina, Centro de Ciências da Educação). Santa Catarina, SC.

Marcondes, C. B. (2006). Terapia larval de lesões de pele causadas por diabetes e outras doenças. Revista Do Instituto De Medicina Tropical De São Paulo, 48(6), 320-320.

Marconi, M. \& Lakatos, E. M. (2017). Técnicas de Pesquisa. (8a ed.), Atlas.

Marques, A. C. T. L., \& Marandino, M. (2018). Alfabetização científica, criança e espaços de educação não formal: diálogos possíveis. Educação e Pesquisa, 44.

Matos, C. H. C., de Oliveira, C. R. F., de França Santos, M. P., \& Ferraz, C. S. (2009). Utilização de modelos didáticos no ensino de entomologia. Revista de biologia e ciências da terra, 9(1), 19-23.

Oliveira, S. G. (2017). A alfabetização científica no ensino fundamental: desafios encontrados pelos docentes em escolas municipais de Ilhéus-Bahia. Dissertação (Mestrado) - Universidade Estadual de Santa Cruz. Programa de Pós-Graduação em Educação em Ciências. Ilhéus, BA.

Pacheco, P. C., \& Pereira, R. F. S. (2021). Aspectos históricos, aplicações parasitárias e coleta em entomologia forense. Revista Multidisciplinar em Saúde, $2(1), 41-41$

Papavero, N. (1994). Fundamentos práticos de taxonomia zoológica. Editora Unesp.

Pereira, A. M. (2014). A contribuição do uso da tecnologia no ensino de ciências para alunos do sétimo ano da rede estadual do município de Ibaiti. Monografia. Pós Graduação em Ensino de Ciências - Pólo de Ibaiti, Universidade Tecnológica Federal do Paraná - UTFPR.

Santos, D. C. J., \& de Souto, L. S. (2011). Coleção entomológica como ferramenta facilitadora para a aprendizagem de Ciências no ensino fundamental. Scientia plena, $7(5)$.

Santos, M., da Silva, R. A., \& Antunes, S. C. (2018). Artrópodes. Revista de Ciência Elementar, 6(2).

Santos, P. R. C., de Almeida Silva, J. O., Aragão, V. L., da Rocha, M. F. C., \& Nascimento, R. F. O. (2021). Coleção didática zoológica: divulgação científica e auxílio para o ensino e aprendizagem de Ciências. Experiências em Ensino de Ciências, 16(1), 656-669.

Sasseron, L. H. (2015). Alfabetización científica, enseñanza por investigación y argumentación: relaciones entre las ciencias de la naturaleza y la escuela. Ensaio Pesquisa em Educação em Ciências (Belo Horizonte), 17(spe), 49-67.

Tonini, L., Sarmento-Soares, L. M., Roldi, M. M. C., \& Lopes, M. M. (2016). A coleção didática de peixes no Instituto Nacional da Mata Atlântica (INMA), Santa Teresa, Espírito Santo, Brasil: subsídios para o Ensino de Zoologia. Bol. Mus. Biol. Mello Leitão, 38, $347-362$.

Wegener, M. K., Wesner, C. W., de Moraes, G. P., \& Müller, G. A. (2016). Scientific communication about the distribution of insect boxes in schools: Report on experience. Extensio: Revista Eletrônica de Extensão, 13(24), 198-203. 